\title{
Teleología Naturalizada: Los conceptos de función, aptitud y adaptación en la Teoría de la Selección Natural ${ }^{*}$ \\ (Naturalized Teleology: The concepts of function, fitness and adaptation in Natural Selection Theory)
}

\author{
Gustavo CAPONI
}

Recibido: 3.10.2011

Versión final: 18.11.2011

BIBLID [0495-4548 (2013) 28: 76; pp. 97-114]

RESUMEN: En la Teoría de la Selección Natural, el concepto de función biológica debe suponerse para delimitar el concepto de aptitud; y éste debe suponerse para delimitar el concepto de adaptación y también para explicar el fenómeno al que este último alude. Esos tres conceptos, por otra parte, son especificaciones de tres conceptos de aplicación más universal. El concepto de función biológica es un caso particular del concepto general de función; y el concepto de aptitud especifica el concepto de eficiencia. El concepto de adaptación, mientras tanto, es una especificación del concepto de diseño. La visualización de esas articulaciones conceptuales permite entender la legitimidad de la naturalización de la teleología operada por el darwinismo.

Palabras clave: adaptación; aptitud; diseño; función; teleología; Teoría de la Selección Natural.

ABSTRACT: In Natural Selection Theory, the concept of biological function must be supposed to elucidate the concept of fitness; and this one must be supposed to elucidate the concept of adaptation and also to explain the phenomenon to which the last one alludes. These three concepts, on the other hand, are specifications of three concepts of more universal application. The concept of biological function is a particular case of the general concept of function; and the concept of fitness is a specification of the concept of efficiency. The concept of adaptation, meanwhile, specifies the concept of design. The visualization of these conceptual joints allows to understanding the legitimacy of the naturalization of the teleology produced by the Darwinism.

Keywords: adaptation; design; fitness; function; teleology; Natural Selection Theory.

Asumiendo la concepción de función como papel causal propuesta por Cummins (1975, 2002), y rechazando, consecuentemente, la llamada concepción etiológica del concepto de función, ${ }^{1}$ en este trabajo analizaré la articulación existente, dentro de la Teoría de la Selec-

* Agradezco a los evaluadores anónimos de THEORIA las observaciones y comentarios realizados sobre la primera versión de este trabajo. Creo que al intentar atenderlas, pude clarificar y mejorar tanto mi planteo del tema como mi argumentación.

${ }^{1}$ Analicé y critiqué la llamada 'concepción etiológica del concepto de función' en varios trabajos previos, en los que también intenté convalidar una versión particular de la posición de Cummins (Cf. Caponi: 2002a; 2010a; 2010b; 2010c). Creo, básicamente, que ese modo de entender el concepto de función presenta una dificultad crucial: la de desconocer contextos del discurso biológico actual, por ejemplo la Fisiología y la Autoecología, en los cuales las imputaciones funcionales no conllevan ningún compromiso con hipótesis evolucionistas (Caponi 2010a, 57). Por otra parte, la propia polaridad entre función propia y función accidental (Millikan, 1989), ya implica el reconocimiento tácito de la existencia de imputaciones funcionales no fundamentadas en explicaciones evolutivas; en caso contrario, no se podría hablar de funciones accidentales como las desempeñadas por las exaptaciones (Caponi 2010a, 56). La tesis etiológica es, en suma, innecesariamente restrictiva: ella deslegitima muchos usos habituales, y no problemáticos, del concepto de función. Su error de base, sin embargo, no reside tan- 
ción Natural, entre los conceptos de función biológica, aptitud y adaptación. En dicha teoría, estos conceptos, según espero mostrar, se articulan de la siguiente manera: el concepto de función biológica debe suponerse para entender el concepto de aptitud; y éste debe suponerse tanto para elucidar el concepto de adaptación, como para explicar el fenómeno al que este último alude. Sin embargo, para entender esos tres conceptos, y su articulación, será necesario presentarlos como especificaciones de tres conceptos de aplicación más universal.

El concepto de función biológica será presentado como un caso particular del concepto general de función, entendido más o menos à la Cummins; y el de aptitud será entendido como una especificación del concepto de eficiencia. Por su parte, el concepto de adaptación será presentado como una especificación del concepto de diseño. Estos tres últimos conceptos, diré también, guardan entre sí relaciones análogas a las que afirmo guardan los conceptos de función biológica, aptitud y adaptación. El concepto general de función debe ser presupuesto para entender el de eficiencia; y éste debe asumirse para delimitar el de diseño y para explicar la existencia de objetos diseñados. En ambas series, por otra parte, hay implicado un ascenso epistemológico que arranca en el mero análisis de un proceso, que sería lo que ocurre en las atribuciones funcionales (Cummins 1975, 765), que pasa luego por un juicio comparativo, como el implicado en las nociones de eficiencia y de aptitud; y que llega, finalmente a la explicación histórica supuesta en las nociones de diseño y de adaptación. Del análisis a la comparación; y de ésta a la historia: he ahí, en síntesis, la concatenación que se da entre las nociones que aquí hemos de examinar.

Ese análisis conceptual, que sería el objetivo inmediato del trabajo, responde, empero, a un objetivo mediato y más ambicioso: ratificar el carácter legítimamente teleológico de esos tres conceptos fundamentales de la Biología Evolucionaria a los que me estoy refiriendo. Es decir: ellos son efectivamente de cuño teleológico; pero la teleología por ellos implicada es perfectamente compatible con un entendimiento científico, y si se quiere materialista, de los fenómenos biológicos. En este sentido, mi posición se aproxima de la de autores como Ayala $(2004,52)$ y Lennox $(1993,419)$, que han sabido reconocer el carácter legítimamente teleológico de la explicación seleccional darwiniana, y se aleja de la posición de autores como Mayr $(1961,1504)$ y Ghiselin $(1997$, 294), que han pretendido negarlo. Concuerdo con Elliott Sober $(1993,82)$ en el hecho de que el darwinismo permite, y conlleva, una genuina naturalización de la teleología; y, contrariando a Dawkins $(1996,1)$, me interesa subrayar que esa naturalización no es una reducción de lo aparentemente teleológico a lo mecánico. En este caso, por lo menos, naturalización no es eliminación por reducción (Cf. Ariew 2007, 179).

to ahí, sino más bien en la superposición entre los conceptos de adaptación y función (Caponi, 2010b, 78). Los defensores de la concepción etiológica no perciben, justamente, lo que aquí quiero mostrar: las explicaciones seleccionales de la adaptación, lejos de justificar las imputaciones funcionales, las presuponen. En Lewens (2007), Garson (2008) y Gayon (2010) pueden encontrarse excelentes contraposiciones entre concepciones etiológicas y concepciones sistémicas del concepto de función.

Theoria 76 (2013): 97-114 


\section{Diseño, función y eficiencia}

No debemos temerle a la palabra 'diseño'. Ella es moneda corriente en el discurso de la Biología normal (Cf. Wainwright et. al. 1980, Weibel et. al. 1998); y en español, por lo menos, su significado no se confunde con el de 'designio' (Cf. Caponi 2010b, 90 n.4). Usado de un modo adecuado, comprendiendo correctamente el concepto por él expresado, ese vocablo no tiene por qué llevarnos a naufragar en las aguas de la Teología; y para usarlo tampoco es necesario escudarse, como lo hace Ruse (2003, 266; $2008,91)$, en la disculpa de la metáfora inevitable. ${ }^{2}$ Es importante, sin embargo, que se pueda dar una caracterización precisa, pero al mismo tiempo general y no intencional, de lo que hemos de entender por 'objeto diseñado'; y creo que ella podría ser la siguiente: $X$ es un objeto diseñado en la medida en que alguno de sus perfiles sea el resultado de un proceso de cambio direccionado por incrementos en la eficiencia con la que ese perfil cumple una función (Cf. Caponi 2010b, 93).

No hay, lo subrayo, ningún riesgo de circularidad en el hecho de recurrir a la noción de función para clarificar la de diseño. Eso sería así, si a la manera de Kitcher, definimos función a partir de diseño. Para él, "las entidades tienen funciones cuando están diseñadas para hacer algo" (Kitcher 1998, 492); y ese algo es, justamente, lo que asumimos como función (Kitcher, 1998, 479 y 492). Pero Kitcher se ve forzado a eso porque él acepta esa concepción etiológica del concepto de función (Cf. Kitcher 1998, 494) que ya dije haber rechazado. Para Kitcher $(1998,495)$, como para Ruth Millikan (1984, p.72), una imputación funcional supone el conocimiento de un proceso de diseño, guiado intencionalmente, o motorizado por la selección natural (Cf. Krohs, 2009, 72). No estoy diciendo, y lo aclaro, que la concepción etiológica de función sea circular: mi rechazo a ella, como ya dije en la nota 1 , tiene que ver con otras razones. Lo que quiero indicar aquí es que, si se evita esa concepción, se puede delimitar el concepto de diseño sin incurrir en la circularidad que supondría el recurso a una concepción del concepto de función que, como la etiológica, ya presupone a la propia idea de diseño (Cf. Krohs 2009, 72-3).

Pienso, en efecto, que asumiendo una posición como la de Cummins, ese recurso a la noción de diseño para caracterizar la de función puede evitarse, afirmándose que una función es, simplemente, el papel causal que algo cumple en el desarrollo de cualquier proceso o en el modo de operar de cualquier sistema (Cf. Caponi, 2010a). Según este modo de entender el concepto de función, lo asumo y lo subrayo, cualquier proceso causal puede ser analizado funcionalmente, imputando a cada uno de sus elementos un papel causal en su desarrollo; y a ese respecto, la posición de Margarita Ponce $(1987,106)$ me parece la más correcta y coherente: según ella, en un análisis funcional, la entidad funcional es "el fenómeno o el hecho que comprendemos en virtud de sus consecuencias en cada caso de explicación; y la función es el efecto de la cosa funcional que contribuye a

\footnotetext{
${ }^{2}$ En inglés, donde el término 'design' expresa tanto lo que nosotros entendemos por 'designio', como lo que entendemos por 'diseño', el uso de esa expresión parece no poder dejar de despertar reticencias (Cf. Reiss 2009, 21). Lo cierto, sin embargo, es que tampoco en inglés él deja de ser usado en el sentido de 'diseño'; que es la única acepción que aquí nos interesará.
} 
la consecución del estado de cosas o del fenómeno por cuyas causas inquirimos en ese mismo proceso explicativo."

Aunque yo preferiría expresar esa misma idea diciendo que, en un análisis funcional, el ítem funcional es simplemente el fenómeno, o elemento, cuya contribución, o intervención, en la ocurrencia de un proceso particular, nosotros queremos identificar; y la función es la contribución de esa entidad en dicho proceso. Nuestro análisis privilegia un estado de cosas, o un proceso, como merecedor de explicación; y, a partir de ahí, sus condiciones de ocurrencia son pensadas en virtud de su papel causal, de su función, en esa misma ocurrencia (Cf. Davies 2001, 182). Asumo, por eso, que la caracterización del concepto de función aquí propuesta es todavía más amplia que aquella que algunos defensores de la perspectiva de Cummins suelen sostener.

Tal el caso de John Reiss $(2009,358)$, que define 'función' como "efecto de una parte (órgano, conducta, etc.) que es condición para la existencia continuada del sistema al que esa parte pertenece". En este sentido vuelvo a coincidir con Ponce (1987, 103 y 106): la idea de función, según la usamos en muchos contextos, no está necesariamente comprometida con la persistencia del ítem funcional, ni tampoco con la regularidad o persistencia del sistema o proceso dentro del cual ese ítem opera. Se puede hablar, y de hecho hablamos, de la función, del papel causal, de un evento determinado en la ocurrencia de un proceso único (Cf. Caponi 2010a, 79-80). Hablamos, por ejemplo, de la función que la succión de una chapa caída en la pista de despegue de un aeropuerto, pudo haber tenido en una catástrofe aeronáutica; y es atendiendo a esa posibilidad del lenguaje funcional que prefiero hablar de una idea procesal, más que sistémica u organizacional, de función: siempre que exista un proceso causal, se puede imputar una función, un papel causal, a cualquiera de los elementos que efectivamente intervienen, causan, su ocurrencia.

Donde haya explicaciones causales, podríamos así decir, habrá siempre análisis y atribuciones funcionales posibles; porque esos análisis y esas imputaciones, como de algún modo también lo dice Ponce $(1987,103)$, no son más que el reverso de esas explicaciones y atribuciones causales (Davies 2001, 85). Pero, aunque esta caracterización del concepto función sea sumamente amplia y tolerante, el concepto de objeto diseñado que puede delinearse a partir de ella, no lo es. Aunque todo proceso causal, y todo sistema, pueda ser funcionalmente analizado, sólo de algunos sistemas muy particulares puede decirse que han estado sometidos a procesos de cambio direccionados por incrementos progresivos en la eficiencia con la que algunos de sus elementos cumplen un papel en su funcionamiento. Cualquier proceso causal puede ser analizado funcionalmente, imputando a cada uno de sus elementos un papel causal en su desarrollo; pero, de la idea de diseño que presenté más arriba, ya se deduce que el simple hecho que una entidad cumpla una función, e incluso el hecho que ella resulte adecuada para cumplirla, es insuficiente para considerar que esa entidad integre un objeto diseñado, o que ella sea un objeto de esa condición.

La luna cumple una función en el movimiento de las mareas: ella tiene un papel causal en ese proceso. Pero nada en ella se modificó en virtud de un mejor cumplimiento de dicha función; ni tampoco ella esta ahí en virtud de esa función. Regular el movimiento de las mareas no es la razón de ser de la luna; por eso no la consideramos 
como un objeto diseñado, ni tampoco consideramos que los sistemas luna-masa de agua lo sean. En realidad, la identificación entre función y razón de ser es el error que está en la base de la llamada concepción etiológica del concepto de función. La idea de razón de ser sólo es aplicable cuando se alude a componentes de objetos diseñados: algunos perfiles de éstos, justamente los que permiten caracterizarlos como diseñados, tienen una razón de ser en tanto y en cuanto ellos obedecen a un proceso direccionado por el incrementó, o la optimización, de su desempeño funcional.

Nótese, además, que la noción de eficiencia también es anterior a la de diseño; y es por eso que aquélla puede invocarse para clarificar a esta última noción. No ocurre lo mismo, sin embargo, con la noción de función: ella es anterior a la de diseño, pero la noción de eficiencia la supone. Quiero decir: precisamos de la noción de función para entender la de eficiencia; y de estas dos para, posteriormente, entender la de diseño. Aunque escogiendo piedritas para hacerlas rebotar en la superficie de un estanque, constatemos que las aplanadas son más eficientes en el cumplimiento de esa función que las más esféricas, tampoco diremos que aquellas son objetos diseñados: sus perfiles fueron modificados por agentes físicos que nada tenían que ver con el cumplimiento de esa u otra función. Se dirá, claro, que en ese ejemplo, la eficiencia de las piedras aplanadas se juzga no sólo en virtud de la mera referencia a una función, sino que ahí está también presupuesta la referencia a un proyecto de uso, que es, justamente, el de producir rebotes. Pero eso no puede llevarnos a decir que esas piedras, moldeadas por la simple acción de las intemperies, sean objetos diseñados.

Creo, por otra parte, que se puede considerar a la noción de eficiencia como un caso especial de la noción de efectividad. En lugar de decir que las piedras aplanadas son más eficientes para rebotar que las arredondeadas, podemos simplemente decir que ellas son más efectivas que las arredondeadas en el cumplimiento de esa función; sin que eso conlleve ninguna pérdida de información en lo atinente al papel causal que la forma plana puede tener en la producción de esos rebotes. Lo único que quizá se haya perdido es la indicación de que dicha efectividad resulta acorde con un proyecto de uso; y esa referencia a un proyecto de uso, manifestada en ese juicio sobre la eficiencia de las piedras aplanadas, parece contrariar la alegación de que la noción biológica de aptitud pueda ser considerada como un caso especial de la noción de eficiencia. Pero, cuando más adelante analicemos esa noción de función biológica que, según también ya dije, es un caso especial de la noción procesal de función que acabo de enunciar, veremos que existe un equivalente, o un análogo biológico, fácilmente reconocible y aceptable, de esa referencia a un proyecto de uso que se manifiesta en el caso de nuestra evaluación de las piedritas aplanadas.

Por ahora, déjeseme simplemente apuntar que lo que ocurre con las piedras aplanadas y su propensión a rebotar en el agua, es algo semejante a lo que ocurre cuando, buscando un material que sea un buen conductor térmico, decimos que, para el cumplimiento de esa función, el hierro resulta más eficiente que el plomo. Lo que estamos diciendo sobre esos materiales es simplemente que el hierro es un conductor térmico más eficiente, más efectivo, que el plomo. El hierro cumple esa función, ese papel causal, más rápidamente, y con una perdida de calor menor, que el plomo. Pero eso 
también lo diríamos si estuviésemos buscando un material maleable que transmita el calor más lentamente que el hierro.

Diríamos que el plomo es un conductor menos eficiente, menos efectivo, que el hierro; pero justamente por eso lo preferiríamos como material aislante. Sin que eso implique que esos elementos químicos fueron diseñados para ser conductores más o menos eficientes del calor. El coeficiente de conductividad térmica de los metales mide su eficiencia en el cumplimiento de la función, del papel causal, que ellos pueden cumplir como transmisores del calor; pero eso no implica que ellos sean materiales diseñados para ese desempeño (Cf. Caponi, 2010c, p.356). Como sí pueden serlo, en cambio, los artefactos que con ellos construyamos en vistas al cumplimiento de esa función; o como también pueden serlo las aleaciones artificiales que puedan producirse para conseguir un coeficiente predeterminado, mayor o menor, de conductividad.

Lo que la referencia a un coeficiente de conductividad térmica deja también claro, por otra parte, es el carácter intrínsecamente comparativo de las nociones de eficiencia y de efectividad. Juzgar algo como siendo eficiente para una determinado desempeño funcional implica un reconocimiento tácito de que, en lugar de ese elemento juzgado eficiente, podría haber otro menos efectivo en ese desempeño funcional. O implica reconocer que ese elemento podría tener una configuración distinta de la que tiene; y que, en ese caso, sería menos eficiente de lo que es. Pero pese a lo obvio que esto último pueda resultarnos, creo que todavía importa apuntarlo para resaltar la diferencia existente entre el carácter eminentemente comparativo de los juicios de eficiencia y el carácter meramente analítico de las atribuciones funcionales.

$\mathrm{Al}$ atribuirle una función a un elemento dentro de un sistema o proceso, sólo aludimos a ese elemento y apuntemos su papel causal dentro de ese sistema o proceso; por eso se puede decir que se trata de una operación de análisis: se habla, clásicamente, de análisis funcional (Hempel 1979 [1959]; Cummins 1975). Atribuir funciones, hasta está de más decirlo, no es trazar comparaciones, tácitas o implícitas, sobre cuestiones de eficiencia o efectividad; y eso nos permite entrever una escala de complejidad epistemológica creciente que va desde las meras atribuciones causales a las funcionales, de éstas a las comparaciones de eficiencia, o efectividad, llegando por fin hasta las atribuciones de diseño que son de naturaleza eminentemente histórica.

Pasar de una atribución causal a una funcional ya es ir de una relación binaria a una relación ternaria: la relación de causación es una relación entre dos términos, la causa y su efecto; la relación funcional, en cambio, es una relación entre tres términos: el ítem funcional, su efecto dicho funcional, y el proceso dentro del cual ese ítem funcional desempeña ese papel causal, o función, que le atribuimos. Mientras tanto, las ideas de eficiencia y efectividad suponen comparaciones, y diferencias, de desempeño funcional entre formas alternativas, posibles o efectivas, de esos ítems funcionales; y la idea de diseño supone que hay sistemas que se configuraron, que se modificaron, en virtud de esas diferencias.

\section{Procesos de diseño}

Lo dicho hasta aquí también permite delinear una caracterización de lo qué es un proceso de diseño: un proceso orientado por la detección y refuerzo de la efectividad, o 
eficiencia, del desempeño funcional de un elemento al interior de un sistema. La erosión hídrica que modela los cantos rodados haciéndolos progresivamente más eficientes como proyectiles no es un proceso de diseño. Dicha erosión es insensible a ese incremento en el posible desempeño de las piedras como proyectiles, y tampoco se orienta por su progresiva acentuación. Como tampoco es un proceso de diseño el incremento de la humedad del aire que aumenta su capacidad de conducir descargas eléctricas. El aire funciona como conductor de esas descargas, y el incremento de la humedad aumenta esa conductividad. Pero, dicho incremento de la humedad no estuvo ni pautado, ni ordenado, ni direccionado, ni causado, bajo ningún punto de vista, por ese aumento de la eficiencia del aire en el desempeño de su habitual función conductora.

En un proceso de diseño la eficiencia tiene que contar del lado de las causas, y no sólo del lado de los efectos. La eficiencia no sólo debe incrementarse como resultado de ese proceso, ese incremento de eficiencia debe pesar, debe poder contar, como un factor rector de ese proceso. La dirección de éste, como ocurre en las modificaciones de caracteres propiciadas por la selección natural, debe estar canalizada por esos incrementos de eficiencia. Cosa que no ocurriría, por ejemplo, en el caso de guijarros, que al ser arrastrados por un río, se transforman en factores erosivos; y, por el desempeño de esa misma función erosiva, que los lleva a desgastarse por el rozamiento contra otros materiales, acaban cobrando contornos y tamaños que incrementan dicha capacidad erosiva. En este caso, no menos que en el caso de las piedritas que devinieron buenas rebotadoras, ese incremento en la capacidad de erosionar no rigió, no pautó, bajo ningún punto de vista, el empuje de las aguas y el rozamiento de esos guijarros contra los otros materiales. Este proceso fue insensible a ese incremento de capacidad erosiva que él mismo retroalimentaba; y por eso, en este caso, también puede decirse que el incremento de esa eficacia funcional quedó sólo del lado de los efectos.

El tallado de una piedra, ejecutado por un hombre para hacer un hacha, sí es, en cambio, un proceso de diseño. El tallador escoge perfiles de la piedra que puedan prestarse a la forma y uso que él quiere darle a esa herramienta, y los va modelando para adecuarlos a dicho uso; tornándolos eficientes en el corte. Pero, aunque eso sea un proceso de diseño, la producción accidental de lascas que se desprenden de la piedra que está siendo tallada, no lo es. Aun cuando esas lascas puedan ser convenientes para hacer punzones con los cuales zurcir el cuero. Es decir: la piedra con la que se inició todo el proceso, aunque seguramente fue escogida por su forma conveniente, no es un objeto diseñado. Ella no es resultado de un proceso de diseño. Como tampoco lo son las lascas desprendidas de ella durante el tallado. Aunque éstas, luego y accidentalmente, también se muestren adecuados para la manufactura de otras herramientas.

El martillar del tallador sobre la piedra es un proceso de diseño en lo atinente al hacha; pero no lo es en lo atinente a las lascas: con relación a éstas, el martillar es semejante a la acción del agua sobre los cantos rodados. Como ya dije, cuando la obtención, o el incremento, de un desempeño funcional se encuentra sólo del lado de los efectos de un proceso, éste no es un proceso de diseño; para que lo sea es necesario que, de alguna forma, ese incremento esté del lado de las causas que direccionan ese 
proceso. Eso lo vemos en lo que suele ocurrir con nuestros zapatos después de andarlos un año. El uso, paulatinamente, produce modificaciones en ellos que, en general, los hacen más confortables; pero nadie dirá que esos cambios son mejoras de diseño: ellos sólo son deformaciones resultantes de un proceso totalmente ajeno a esa eventual mejora funcional. El uso, aunque ocasionalmente mejora la funcionalidad de los útiles, e incluso de los órganos, no es un proceso de diseño; a no ser, claro, que dicho uso, como ocurre en el entrenamiento deportivo, ya esté ordenado en vistas a producir incrementos de desempeño funcional.

Las deformaciones de los zapatos viejos, y los callos que empiezan a protegernos las manos después de trabajar un tiempo como remeros, son cosas muy convenientes; pero no resultan de un proceso de diseño. Como sí ocurre son la mayor parte de las características de un nuevo par de Nikes, o como lo son los caracteres de los seres vivos que resultan de la selección natural. Lo primero, claro, es obvio; lo último, lo sé, resulta dudoso: quizá una metáfora arriesgada, o un abuso de lenguaje. Pero si miramos bien las cosas, veremos que en ambos casos estamos suponiendo la intervención de procesos que no solamente incrementaron el desempeño funcional de ciertas estructuras, sino que además fueron causadas por ese incremento. Aunque de dos modos diferentes, ambos procesos, el del diseño de las zapatillas y el de la selección natural, fueron causados, motorizados, por ese incremento progresivo en el desempeño funcional, o eficiencia, de las estructuras a ellos sometidas.

En el caso de los artefactos, las funciones en cuestión son los papeles que esos objetos cumplen en los procesos para cuya realización ellos fueron construidos o adoptados; y así, de la misma forma en que diremos que las Nikes fueron diseñadas para $b a$ cernos felices, también diremos que un hacha es un objeto diseñado en la medida en que sus perfiles fueron modificados para mejor cumplir con la función de cortar o golpear. O diremos que una semilla cultivable es un objeto diseñado porque sus perfiles responden a un proceso de hibridación, de selección artificial, o de manipulación genéti$\mathrm{ca}$, tendiente a incrementar su rendimiento en un determinado suelo o su resistencia a una plaga.

Mientras tanto, en el caso de seres vivos no sometidos a ninguno de estos últimos procesos, diremos que ellos están diseñados porque sus perfiles se modificaron, por selección natural, en virtud de que dichas modificaciones permitían el mejor cumplimiento de alguna función biológica; y por función biológica no debemos entender otra cosa que el papel causal que las estructuras o fenómenos orgánicos cumplen en la realización del ciclo vital de un determinado organismo (Caponi 2010b, 83). El acabamiento de la ontogenia de un ser vivo, el reaseguro de su propia pervivencia, y el dar lugar a una progenie que también sea capaz de desarrollarse, de reproducirse y de pervivir, son notas que configuran lo que quiero indicar aquí con la expresión 'ciclo vital'; y ellas, permitiéndonos delimitar la noción de función biológica, también nos permiten comprender la noción biológica de eficiencia sin tener que apelar a la idea de un proyecto de uso. Será eso, además, lo que nos conducirá, sin mayores dificultades, hasta la noción de diseño biológico. 


\section{La selección natural como proceso de diseño}

La noción biológica de función es un caso particular, la aplicación a un dominio restringido, de la noción sistémica, o procesal, de función. Es una especificación de esta última, que es inherente a las ciencias biológicas en general (Cf. Caponi, 2002a, p.68). Ella se basa en el reconocimiento de que la realización del ciclo vital es la meta intrínseca, e incluso definitoria, del ser vivo (Caponi 2010a, 62); y es claro que no puedo arrogarme ninguna originalidad al formularla. Ella, sin ir más lejos, ya consta, palabras más, palabras menos, en el viejo diccionario de Abercrombie, Hickman y Johnson (1961, 108). Según estos autores, en Biología, el termino 'función' designa "la función de una parte del organismo en el sentido en que esa parte coadyuva en el mantenimiento de la vida y en la capacidad de reproducción" (Cf. Caponi 2010a, 63). Pero, si quiere usarse el lenguaje de Cummins $(1975,762)$, puede también decirse que la realización del ciclo vital es, en última instancia, la capacidad sistémica que delimita al concepto de función biológica, curándolo de toda promiscuidad (Caponi, 2010a, 63) y otorgándole, al mismo tiempo, una cierta dimensión normativa (Cf. Caponi, 2010a, 66; Jaume 2011, 108).

Asumiendo esto que acabo de decir, cabe afirmar que la aceptación de una imputación funcional del tipo $Y$ es la función biológica de $X$ en $Z$, supone: [1] que $Z$ es el ciclo vital de un ser vivo; [2] que $X$ produce $Y$; y [3] que $Y$ tiene un papel causal en la realización de $Z$. Así, todo $X$, tal que tenga un efecto $Y$ que contribuya para que un organismo se preserve, se desarrolle y se reproduzca en un ambiente determinado, podrá ser descrito como poseyendo una función biológica; y ésta no será otra que la contribución de $X$ al cumplimiento de esa preservación, de ese desarrollo y de esa reproducción (Cf. Caponi 2010b, 79 y ss). Siendo esa referencia al ciclo vital, que es constitutiva del concepto biológico de función, la que nos permite hacer juicios de eficiencia sin presuponer un proyecto de uso: una conformación morfológica, o un comportamiento, serán mas o menos eficientes en la medida en que contribuyan, de un modo más o menos efectivo, en la realización de ese ciclo vital.

Quiero decir: una vez asumida la idea de función biológica, con esa pequeña pero tan innegable como inevitable carga de teleología que se pone en evidencia al vincularse indisolublemente la imputación funcional con la referencia a una meta o estado privilegiado de los fenómenos organísmicos (Caponi: 2002a, 71-2; 2010a, 64), y una vez aceptada la atribución de una función biológica a una estructura orgánica específica, cabe juzgar la eficiencia de esta última por el simple expediente de comparar su desempeño con el de una estructura ligeramente diferente que esté cumpliendo una función idéntica o semejante a la que ella desempeña. Aunque también se puede juzgar esa eficiencia considerando, y mostrando, que dicha estructura podría tener un desempeño funcional diferente, mejor o peor en términos biológicos, si ella fuese distinta de cómo es (Cf. Cummins y Roth 2010, 79).

Importa subrayar, además, que en el caso del concepto biológico de función, como ocurre en el caso del concepto general de función, poseer una función tampoco es lo mismo que estar diseñado o que tener una razón de ser. Cuando entra en juego el concepto biológico de función, esa superposición errónea entre ambos conceptos, que como dije más arriba está en la base de las llamadas concepciones etiológicas, cobra la 
forma de una identificación entre el concepto de función y el concepto de adaptación (Caponi 2010a, 59); y es ése primer error el que conduce a homologar, como lo hacen los autores de Diseño mecánico de organismos (Wainwright et. al. 1980, 13), atribuciones funcionales con atribuciones de diseño.

El olor que produce una planta al metabolizar una sustancia tóxica que contamina el terreno en el que ella crece, puede ayudarla a ahuyentar unos insectos que acaban de invadir la región; y entonces podrá decirse que la producción de ese olor tiene una función importante en su ciclo vital. Pero no podremos decir que la capacidad de producir ese olor sea una adaptación. ${ }^{3}$ Ella no surgió como respuesta a una presión selectiva desencadenada por la presencia de esa plaga. En lo que respecta a esa función ella sólo será una mera, aunque oportuna, aptación (Cf. Brandon 1990, 171; Ginnobili 2009, 23, n. 3; Caponi 2011, 73). Luego, claro, si la plaga se torna una presencia constante, y la capacidad de generar ese aroma protector presenta una variabilidad hereditaria tal que hace que algunas plantas sean capaces de producir un aroma más intenso, y más eficiente como repelente de insectos, entonces, en ese caso, sí estaremos ante el surgimiento de una presión selectiva que irá premiando cualquier modificación metabólica hereditaria que incremente dicha capacidad. Esas modificaciones, al ser promovidas por una presión selectiva, podrán ser consideradas adaptaciones.

Soy plenamente conciente, por otra parte, de estar entendiendo el término 'aptación' de un modo un poco diferente a como lo entendieron Gould y Vrba (1998 [1982], 521). Asumiendo la concepción etiológica de función propuesta por George Williams (1966, 261), Gould y Vrba (1998 [1982], 520) superpusieron los conceptos de función y de adaptación. Para ellos sólo era posible atribuirle una función a una estructura biológica en la medida en que la producción del efecto que en ella consideramos funcional, también fuese un efecto seleccionado. Una aptación, en cambio, sería todo aquello cuya operación o presencia es útil o conveniente para un ser vivo (Gould y Vrba 1998 [1982], 521); y una adaptación seria un caso especial de aptación: las adaptaciones son ad-aptaciones, son aptaciones pergeñadas por la selección natural en virtud de su desempeño funcional (Gould y Vrba 1998 [1982], 523). Mientras tanto, las exaptaciones son aptaciones cuyo origen, o bien no obedece a la selección natural, o bien obedece a un proceso selectivo que apuntó a funciones diferentes que las que ahora ellas cumplen; pero que después, en ambos casos, la selección natural coopta para el ejercicio de esas nuevas funciones (Gould y Vrba 1998 [1982], 521).

Por mi parte, rechazando la concepción etiológica del concepto de función y apoyándome en lo que he llamado concepto biológico de función, creo que es más simple llamar aptación a todo aquello que cumple una función biológica; es decir: a todos aquellos caracteres fenotípicos, en sentido extendido (Cf. Dawkins 1999, 293), que contribuyen a la realización del ciclo vital de un ser vivo. Las adaptaciones, mientras tanto, no obstante ese concepto diferente de aptación, pueden ser entendidas de la misma manera en que Gould y Vrba (1998 [1982], 521), al igual que prácticamente to-

\footnotetext{
${ }^{3}$ Nótese que, si se acepta la concepción etiológica, tampoco estaríamos habilitados a decir que ese efecto repelente tenga cualquier función. Eso ocurre por la ya apuntada superposición entre los conceptos de función y adaptación en la que incurren los defensores de esa tesis.
} 
dos los evolucionistas (Cf. West-Eberhard 1998, 8; Griffiths 1999, 3), lo hacen: como estructuras que evolucionaron, por selección natural, en virtud de su desempeño funcional. Las exaptaciones, por lo demás, también pueden continuar siendo entendidas de modo análogo a como Gould y Vrba (1998 [1982], 521) lo proponen. En síntesis: aptación es todo aquello que desempeña una función biológica; y ad-aptación es únicamente aquello que fue seleccionado y modificado para hacerlo de un modo más eficiente. Siendo ahí que puede establecerse la identificación entre adaptación y diseño natural.

Cabe afirmar que un perfil orgánico es un perfil [naturalmente] diseñado, se puede decir que él es una adaptación, si y sólo si, él fue modificado por selección natural en virtud de que dicha modificación permitía un cumplimiento más efectivo de alguna función biológica. Es decir: se puede caracterizar a una estructura orgánica $X$ como estando [naturalmente] diseñada para hacer $Y$, si y sólo si, se cumplen las siguientes condiciones: [1] $Y$ es una función biológica de $X, \mathrm{y}$ [2] $X$ es el resultado de un proceso de cambio producido por la selección natural en virtud de que $X$ haya sido mas eficiente en la realización de $Y$ que sus variantes alternativas (Cf. Allen y Bekoff 1998, 578, Caponi 2010b, 94).

Así, si puede establecerse que algunos de los perfiles de una estructura orgánica exhiben estados de caracteres que fueron seleccionados en virtud del mejor desempeño en el cumplimiento de alguna función relevante para el ciclo vital de sus portadores, entonces ahí sí podremos decir que esa estructura es una estructura diseñada en razón del cumplimiento de dicha función biológica. Por eso, aunque una estructura orgánica cualquiera desempeñe una función relevante en la realización del ciclo vital de un determinado organismo, si ella no exhibe un carácter seleccionado para el cumplimiento de esa función, o si sus perfiles no fueron modificados en virtud de ese desempeño, no podremos decir que ella esté diseñada para cumplirla. No podremos decir que ella es una ad-aptación para esa función. Ella será sólo una aptación, por eficiente que ella sea y por mucho que ella contribuya, incluso, al éxito reproductivo de sus portadores. Estar diseñado, ser una adaptación, no es lo mismo que ser adecuado e eficiente para el cumplimiento de una función. Estar diseñado, ser una adaptación, implica haber sido modificado o construido en virtud de ese cumplimiento (Cf. Sober 1993, 83-4).

Por eso puede decirse que las estructuras vestigiales que hoy no cumplen ninguna función, también fueron diseñadas y merecen ser rotuladas como adaptaciones (Sober 1993, 84). Tal el caso de la posesión del apéndice intestinal en Homo sapiens. Este carácter puede ser considerado una adaptación, aun cuando concluyamos que dichos apéndices ya no cumplen, quizá desde hace mucho tiempo, ninguna función biológica relevante (Cf. Sterelny y Griffiths 1999, 217-8). Esa estructura cumplía una función en el pasado de nuestro linaje, y su forma evolucionó en virtud de esa función. Por eso se puede decir que ella está diseñada, como también decimos que un puñal reducido a modesto cortapapeles está, pese a todo, diseñado para matar.

Nótese, además, que la idea de adaptación que estoy suponiendo aquí se corresponde perfectamente con la definición que de ese concepto propone Douglas Futuyma (1998) en el glosario de Evolutionary Biology: "un proceso de cambio genético en una 
población, debido a la selección natural, por el cual el estado medio de un carácter es perfeccionado en relación a una función específica". Aquí el término es tomado como significando el proceso selectivo que conduce al estado de carácter que también llamamos adaptación; esto último sería la adaptación como resultado. Pero el modo en que ese proceso es caracterizado, confirma la posibilidad de entender a la selección natural como un proceso de diseño: dada una función, si el estado medio de un carácter en una población evolucionó en virtud de su optimización en ese desempeño, entonces puede decirse que ese estado de carácter es una adaptación; y es por lo mismo que puede decirse que ese estado es resultado de un proceso de diseño.

La selección natural es, en efecto, un proceso de diseño (Cf. Dennett 1995, 187 y ss; Caponi 2002b, 15 y ss): ella va modificando los caracteres de los diferentes linajes de seres vivos siguiendo, de una manera oportunista y puntillosa, la senda que le marcan pequeñas, o no tan pequeñas, diferencias e incrementos del desempeño funcional de las distintas partes y pautas comportamentales de dichos seres. La selección natural registra y acentúa, por acumulación, dichas diferencias en el cumplimento de lo que aquí llamé funciones biológicas; y ella sólo actúa en virtud de dichas diferencias. Dennett $(1995,185)$ ha dicho, por eso, que "el trabajo hecho por la selección natural es Investigación \& Desarrollo"; y Ayala $(2004,52)$ usó la feliz fórmula "diseño sin diseñador" para referirse al resultado de ese proceso estricta y claramente regido por el incremento de la eficiencia con la que se cumplen diferentes funciones biológicas.

La caracterización de estructura naturalmente diseñada que aquí propongo se asemeja mucho a la propuesta por Allen y Bekoff $(1998,578)$; y es en ella que de hecho me basé para delinear la mía. Según estos autores, podemos caracterizar una estructura $x$ como estando [naturalmente] diseñada para hacer $y$, si y sólo si, se cumplen las siguientes condiciones: [1] $y$ es una función biológica de $x, \mathrm{y}$ [2] $x$ es el resultado de un proceso de cambio (anatómico o comportamental) debido a selección natural que condujo que $x$ sea superior o mejor adaptada a hacer $y$ que sus versiones ancestrales. Pero, diferentemente de lo que ocurre con la propuesta por Allen y Bekoff, mi caracterización de estructura naturalmente diseñada evita el error de presuponer una concepción etiológica del concepto de función (Cf. Allen y Bekoff 1998, 574). Cosa que, como dije más arriba al referirme a la idea de función propuesta por Kitcher, nos haría incurrir en la circularidad: caracterizaríamos el concepto de función apelando, tácita o explícitamente a la idea de diseño; para después caracterizar a esta última apelando al propio concepto de función. El recurso a la noción biológica [procesal] de función nos evita, en cambio, esa dificultad. ${ }^{4}$

Por otra parte, al caracterizar a la selección natural como siendo un proceso de diseño direccionado por incrementos localmente progresivos en el desempeño funcional

\footnotetext{
${ }^{4}$ Aunque otra forma de ver la cuestión podría ser la siguiente: implícita y vagamente, los defensores de la concepción etiológica estarían cometiendo el error de asumir el concepto, relativamente complejo y algo problemático, de diseño biológico, como si este fuese un concepto primitivo en base al cual cabría delimitar el concepto, más simple y mucho menos problemático, de función. Si esto es así, creo que la quinta regla de Descartes (1983 [1701], 165), me asiste para decir que la estrategia aquí seguida, que va de lo simple a lo complejo, y de lo poco cuestionado a la más cuestionado, parece ciertamente más indicada, que la esbozada por Kitcher o Millikan.
} 
de una estructura, también se está suponiendo (como ocurría con las caracterizaciones generales de objeto diseñado y proceso de diseño propuestas más arriba), una idea de eficiencia en el cumplimiento de una función que tampoco deja de ser anterior a [e independiente de] la propia noción de diseño. Podemos decir que la sustancia olorosa producida por una planta al metabolizar una sustancia tóxica es más eficiente, como repelente de insectos, que la producida por otra planta que crece en la misma región; sin que eso conlleve necesariamente la suposición de que el efecto protector de esa excreción haya sido premiado o acentuado por la selección natural.

De hecho, para que la propia selección natural pueda ocurrir, es necesario que a ella preexistan diferencias de eficiencia en el modo en el que distintas variantes heredables de una estructura al interior de una población, permiten desempeñar una determinada función (Cf. Ginnobili 2009, 14; Caponi 2010b, 88). Por eso, para que las explicaciones seleccionales puedan ser construidas, es necesario que esas diferencias sean previamente conocidas. Las imputaciones funcionales y las comparaciones de eficiencia preexisten a las explicaciones seleccionales; y es justamente el concepto de función biológica, como dije más arriba, el que nos permite caracterizar y sopesar esa eficiencia sin hacer referencia a un proyecto de uso, o a cualquier otra cosa extrínseca a los propios fenómenos biológicos.

\section{Vindicación de la aptitud}

Pero en la Teoría de la Selección Natural ya existe un concepto biológico específico para referirse a esas comparaciones de eficiencia supuestas en las explicaciones seleccionales: el concepto de aptitud. No aludo, lo aclaro, al mero éxito reproductivo diferencial (David y Samadi 2000, 22-3), que es sólo un índice de esa aptitud (Rosenberg 1985, 157; Gayon 1994, 218); sino a la propia capacidad, tampoco la simple probabilidad, de alcanzar ese éxito reproductivo que la variante heredable de un carácter le confiere a sus portadores en un ambiente determinado (Cf. Ginnobili: 2009, 45; 2010, 45). Aludo a eso que Rosenberg $(2006,175)$ denomina 'aptitud ecológica': la menor o mayor capacidad de prosperar y reproducirse en un ambiente determinado que ciertas características heredables pueden otorgarle a un ser vivo. Se trata, para decirlo de otro modo, de la aptitud considerada como concepto causal; y no de los efectos que permiten sopesarla e incluso proyectarla hacia el futuro.

La aptitud ecológica, que Burian $(2005,62)$ rotula relative engineering fitness, debe ser claramente diferenciada, no sólo del suceso reproductivo diferencial ya dado, sino que también debe distinguirse del suceso reproductivo diferencial que puede esperarse de una variante al interior de una población, en un determinado ambiente, en virtud de nuestro conocimiento previo del éxito reproductivo efectivo ya mostrado por esa variante en situaciones análogas. Este último modo de usar el término 'fitness', como Burian (2005, 66-7) apunta, es aquel que ha sido considerado por aquellos autores que han reivindicado lo que se dio en llamar 'concepción propensivista de la aptitud' (Brandon 1978, Mills y Beatty 1979, Sober 2006); y aunque también podemos concordar con Burian $(2005,66)$ en el hecho de que esta acepción del término 'fitness' desempeñó, y aun desempeña, un papel importante en el discurso de la Biología Evolucionaria (Cf. Rosenberg 1994, 124), creo que la idea de aptitud ecológica es la única 
que realmente le confiere utilidad explicativa al concepto de aptitud (Cf. Sober 1993, 69 y 81).

En cambio, en eso Sober (1984, 88 y ss) tiene razón, si se define a la aptitud como mero éxito reproductivo diferencial, esperado o efectivo, ella se transforma en una propiedad causalmente inerte. Ella, como también Endler $(1986,33)$ lo asume, pasa a ser una noción meramente descriptiva y nulamente explicativa. Pero, insisto, si se reconoce lo que Rosenberg $(2006,176)$ llama aptitud ecológica, y se le concede primacía sobre las otras que acepciones del término 'aptitud', esa nulidad explicativa y esa inercia causal desaparecen. Las diferencias de aptitud ecológica que generan las variantes heredables de un rasgo al interior de una población, aunque en general no son nada fáciles de determinar, son motores, y no meros indicadores, de los fenómenos evolutivos (Rosenberg 1985, 157).

El éxito y el fracaso en la lucha por la existencia, según la Teoría de la Selección Natural lo pretende, no dependen del mero azar (Darwin 1859, 127): dependen, en parte, de las características heredables de los individuos que protagonizan esa lucha (Mayr 2001, 116). Dependen de la capacidad que cada uno de ellos tiene de cumplir, de un modo más o menos eficiente, con las funciones biológicas exigidas por las condiciones en las que deben realizar sus ciclos vitales; y es a ese grado de eficiencia que podemos denominar 'aptitud ecológica', o simplemente 'aptitud'. Ésta se puede predicar de variantes de caracteres morfológicos o comportamentales; y así diremos que, en una población $x$ que medra en el ambiente $y$, tal o cual variante resulta más o menos apta en el cumplimiento de tal o cual función. Pero ella también puede predicarse de individuos; y así diremos que, en la población $x$ que medra en el ambiente $y$, los individuos que exhiben tal o cual variante de un carácter, son más o menos aptos que los que no la exhiben.

La aptitud ecológica se dice muchas maneras: de estados de caracteres, de los individuos que los exhiben, y hasta de los factores hereditarios que trasmites dichos estados. Pero lo que aquí más importa es subrayar que, aunque siempre se exigirá que las diferencias en éxito reproductivo corroboren nuestra apreciación sobre la aptitud ecológica de estados de caracteres, de genes, o de individuos, no será el simple conocimiento de esas diferencias lo que podrá citarse como justificación de nuestro juicio. Aunque el mayor éxito reproductivo diferencial sea un índice siempre necesario, y por lo general muy significativo, de aptitud ecológica, él es un criterio insuficiente para establecerla (Rosenberg 2006, 175). Para que esto pueda hacerse es menester conocer las exigencias funcionales a las que esos seres vivos en cuestión están sometidos, y es necesario evaluar correctamente la eficiencia con la que permiten cumplirlas las diferentes formas variantes (Rosenberg y McShea 2008, 55).

Son las diferencias de aptitud ecológica las que explican las diferencias en éxito reproductivo; y es eso lo que significa la spencereana, pero no tan mala, fórmula supervivencia de los más aptos: dada la supervivencia (éxito reproductivo) búsquese la aptitud (ecológica) que la explique; y conjeturada esa aptitud búsquese el éxito reproductivo que la confirme. Por otro lado, y como Brandon $(1990,165)$ de algún modo lo reconoce, la explicación ecológica del hecho que algunas variantes sean más aptas que otras, es un elemento necesario de toda explicación de la adaptación; aunque él se 
equivoque y para decir eso use la palabra 'adaptado' y no 'apto'. Es decir, aunque para referirse a la aptitud ecológica, Brandon use la palabra 'adaptado' en ese mismo sentido a-histórico, que él ya había considerado de poca importancia en la Biología Evolutiva (Brandon 1990, 40). Pero eso es lo que ocurre cuando dejamos que el concepto de aptitud se devalúe, transformándose en sinónimo de lo que sólo debería ser considerado como su manifestación empírica más fácilmente cuantificable.

Brandon $(1990,40)$, que ya había asumido que ser resultado de un proceso selectivo es una nota fundamental del concepto de adaptación, acaba recurriendo a este último para aclarar qué es una explicación por selección natural; y así incurre en una circularidad que hubiese evitado con solamente no renunciar al concepto de aptitud ecológica. Renuncia que, además, también está en la base de la pretendida definición ahistórica de adaptación formulada por Reeve y Sherman (1993, 9): “una adaptación es una variante fenotípica que resulta en la más alta aptitud dentro de un conjunto delimitado de variantes en un ambiente dado" (Cf. Jaume 2011, 104). Estos autores, aun sin saberlo, en realidad estaban definiendo 'aptación'; y para hacerlo correctamente, ellos deberían haber escrito 'éxito reproductivo diferencial' donde simplemente escribieron 'aptitud'. Lo que Reeve y Sherman no vieron es, en definitiva, lo que Brandon tampoco pudo asumir y mostrar con toda la claridad necesaria: el concepto de aptitud es el concepto comparativo (Ariew y Matthen 2002, 56), y a-histórico, que debe usarse para delimitar el concepto eminentemente histórico de adaptación.

Lo que Brandon presenta como una definición de 'adaptedness', de la 'condición de ser adaptativo', es, en realidad, una elucidación del concepto de aptitud ecológica. Brandon $(1990,15)$ dice: " $a$ está mejor adaptado que $b$ en el ambiente $E$, si y solo si, $a$ está mejor preparado que $b$ para sobrevivir y reproducirse en E"; pero, para elucidar el concepto que él realmente estaba intentando elucidar, simplemente podría, y debería, haber dicho que " $a$ es más apto que $b$ en el ambiente $E$, si y solo si, $a$ está mejor preparado que $b$ para sobrevivir y reproducirse en E". En realidad, la aptitud ecológica, la aptitud en su sentido fuerte y originario, explicativo, la aptitud causalmente eficaz, no es otra cosa que eso que Brandon (1990, 14), y otros autores de lengua inglesa, en una opción terminológica infeliz, han llamado 'adaptivennes' o 'adaptedness'. ${ }^{5} \mathrm{Y}$ digo que tal opción es infeliz porque, si la adaptación es resultado de la selección natural, el calificativo 'adaptativo' debería ser reservado para aquello que ha sido seleccionado; dejando que las palabras 'apto' y 'aptitud', sin ser reducidas a meros sinónimos de 'éxito reproductivo', nos presten todos los servicios que nos pueden prestar.

Y en el contexto de nuestra discusión, el mejor servicio que la palabra 'aptitud' nos puede prestar es, justamente, el de designar esa eficiencia heredable que las estructuras orgánicas suelen tener en el cumplimiento de las diferentes funciones biológicas. La aptitud no es simplemente eficiencia, es eficiencia que por su transmisibilidad hereditaria resulta registrable, acumulable y acentuable por la selección natural. Ella, en definitiva, es la que nos permite hablar de la selección natural como proceso de diseño. La aptitud es eficiencia que atraviesa generaciones: es eficiencia que se manifiesta prima-

${ }^{5} \mathrm{Al}$ respecto de esas palabras, de incomoda pero innecesaria traducción al español, ver: Sober $(1993,84)$; Sterelny y Griffiths (1999, 217); y también Huneman $(2011,101)$. 
riamente en el orden de las causas próximas, permitiendo un cumplimiento más efectivo de alguna función biológica; pero, por su condición heredable, ella impacta y se manifiesta en el orden de las causas remotas delineando adaptaciones, generando diseño. Es decir: rigiendo la historia de las formas biológicas en virtud de diferencias e incrementos en su desempeño funcional.

\section{Conclusión}

Dada una noción de contenido teleológico casi imperceptible y por eso difícilmente cuestionable, como lo es la noción de función biológica, se puede acceder a al concepto de aptitud. Esta noción intrínsecamente comparativa designa diferencias heredables de desempeño funcional cuyo reconocimiento sólo nos exige asumir que, dado ese proceso llamado ciclo vital que hemos usado para aclarar la idea de función biológica, se puede también juzgar la eficiencia con la que ciertas estructuras contribuyen causalmente a su realización de una manera que no difiere mucho de lo que ocurre cuando evaluamos la efectividad con la que un metal conduce el calor. Con todo, dada y asumida esa referencia privilegiada al ciclo vital, que es constitutiva de las atribuciones funcionales propias de la Biología, se puede dar un paso más en dirección a un modo teleológico de pensar y decir que las diferencias de aptitud son diferencias de eficiencia.

Así quedamos habilitados para decir que la selección natural, rigiéndose por incrementos en la aptitud de las estructuras biológicas, pautándose por la eficiencia con las que éstas cumplen con las diferentes funciones biológicas, se perfila como un genuino proceso de diseño; cuyos resultados, las adaptaciones, son verdaderos objetos diseñados. Del mero análisis funcional de un proceso natural como es el ciclo vital, pasamos a comparaciones de eficiencia que tienen su correlato objetivo en las diferencias de aptitud; y el reconocimiento del impacto que estas diferencias tienen en la historia de los linajes y de sus caracteres, nos permite decir que la selección natural es un genuino proceso de diseño. He ahí la proeza de Darwin: he ahí la teleología naturalizada.

\section{REFERENCIAS}

Abercrombie, Michael; Hickman, Charles y Johnson, Michael. 1961. Diccionario de Biología. Buenos Aires: Lerú.

Allen, Collin y Bekoff, Mark. 1998. Biological function, adaptation, and natural design. En Nature's purpose: analysis of function and design in Biology, eds. Collin Allen, Mark Bekoff y George Lauder, 571-588. Cambridge, Mass.: MIT Press.

Ariew, André. 2007. Teleology. En Cambridge companion to the Philosophy of Biology, eds. David Hull y Michael Ruse, 160-181. Cambridge, Mass.: Cambridge University Press.

Ariew, André y Matthen, Mohan. 2002. Two ways of thinking about fitness and natural selection. The Journal of Philosophy 99: 55-83.

Ayala, Francisco 2004. In William Paley shadow: Darwin explanation of design. Ludus Vitalis 12: 50-66.

Brandon, Robert. 1978. Adaptation and evolutionary theory. Studies in History and Philosophy of Sciences 9: 181-206.

-. 1990. Adaptation and environment. Princeton: Princeton University Press.

Burian, Richard. 2005. The epistemology of development, evolution and genetics. Cambridge, Mass.: Cambridge University Press. 
Caponi, Gustavo. 2002a. Explicación seleccional y explicación funcional: la teleología en la biología contemporánea. Episteme 14: 57-88.

—. 2002b. La sabiduría de las especies: las poblaciones biológicas como sistemas cognitivos. Ludus Vitalis 18: 9-38.

—. 2010a. Análisis funcionales y explicaciones seleccionales en Biología: una crítica de la concepción etiológica del concepto de función. Ideas y V alores 143: 51-72.

—. 2010b. Función, adaptación y diseño en Biología. Signos Filosóficos 12: 71-101.

—. 2010c. La ciencia de lo sustentable: razón de ser del discurso funcional en Ecología. Principia 14: 349373.

- 2011. La segunda agenda darwiniana: contribución preliminar a una historia del programa adaptacionista. México: Centro de Estudios Filosóficos, Políticos y Sociales Vicente Lombardo Toledano.

Cummins, Robert. 1975. Functional analysis. The Journal of Philosophy 20: 741-765.

- 2002. Neo-teleology. En Functions: new essays in the Philosophy of Psychology and Biology, eds. André Ariew, Robert Cummins y Mark Perlman, 57-172. Oxford: Oxford University Press.

Cummins, Robert y Roth, Martin. 2010. Traits have not evolved to function the way they do because of past advantage. En Contemporary debates in Philosophy of Biology, eds. Francisco Ayala y Robert Arp, 72 88. Oxford: Wiley-Blackwell.

Darwin, Charles. 1859. On the origin of species. London: Murray.

Davies, Paul. 2001. Norms of nature. Cambridge: MIT Press.

David, Patrice y Samadi, Sarah. 2000. La théorie de l'évolution. Paris: Flammarion.

Dawkins, Richard. 1996. The blind watchmaker. New York: Norton.

- 1999. The extended phenotype. Oxford: Oxford University Press.

Dennett, Daniel. 1995. Darwin's dangerous idea. London: Penguin.

Descartes, René. 1983. Reglas para la dirección de la mente [1701]. Buenos Aires: Orbis.

Endler, John. 1986. Natural selection in the wild. Princeton: Princeton University Press.

Futuyma, Douglas. 1998. Evolutionary Biology. Sunderland: Sinaver.

Garson, Justin. 2008. Function and Teleology. En A companion to the Philosophy of Biology, eds. Sahotra Sarkar y Anya Plutynski, 525-549. Malden: Blackwell.

Gayon, Jean. 1994. Examen crítico del concepto de fitness. Signos 8: 213-228.

- 2010. Raisonnement fonctionelle et niveaux d'organization en Biologie. En Les fonctions: des organismes aux artefacts, eds. Jean Gayon y Armand Ricqlès, 125-138. Paris: PUF.

Ghiselin, Michael. 1997. Metaphysics and the origin of species. Albany: SUNY Press.

Ginnobili, Santiago. 2009. Adaptación y función: el papel de los conceptos funcionales en la teoría de la selección natural darwiniana. Ludus Vitalis 17: 3-24.

- 2010. La teoría de la selección natural darwiniana. THEORIA 67: 37-58.

Gould, Stephen y Vrba, Elisabeth. 1998. Exaptation: a missing term in the science of form [1982], en Nature's purpose: analysis of function and design in Biology, eds. Collin Allen; Mark Bekoff y George Lauder, 519-540. Cambridge, Mass.: MIT Press.

Griffiths, Paul. 1999. Adaptation and Adaptationism. En The MIT Encyclopedia of the Cognitive Sciences, eds. Robert Wilson y Frank Keil, 3-4. Cambridge: MIT Press.

Hempel, Carl. 1979. La lógica del análisis funcional [1959]. En La explicación cientifica, Carl Hempel, 295328. Buenos Aires: Paidos.

Huneman, Philippe. 2011. Selection. En Les mondes daminiens, eds. Thomas Heams ; Philippe Huneman; Guillaume Lecointre y Marc Silberstein, 93-148. Paris: Éditions Matériologiques.

Jaume, Andrés. 2011. Adaptabilidad y funcionalidad: una teoría acerca de las funciones biológicas. Ludus Vitalis 19 (35): 97-112.

Kitcher, Philip. 1998. Function and design. En Nature's purpose: analysis of function and design in Biology, eds. Collin Allen, Mark Bekoff y George Lauder, 479-504. Cambridge, Mass.: MIT Press.

Krohs, Ulrich. 2009. Functions as based on a concept of general design. Synthese 166: 69-89.

Lennox, James. 1993. Darwin was a teleologist? Biology \& Philosophy 8: 409-21.

Lewens, Tim. 2007. Functions. En Philosophy of Biology, eds. Mohan Matthen y Christopher Setphens, 525548. Amsterdam: Elsevier.

Mayr, Ernst. 1961. Cause and effect in Biology. Science 134: 1501-1506.

- 2001. What evolution is. New York: Basic Books. 
Millikan, Ruth. 1984: Language, thought and other biological categories. Cambridge, Mass.: MIT Press.

- 1989: In defense of proper function. Philosopby of Science 56: 288-302.

Mills, Susan y Beatty, John. 1979. The propensity interpretation of fitness. Philosophy of Science 46: 263-286.

Ponce, Margarita. 1987. La explicación teleológica. México: UNAM.

Reeve, Hudson y Sherman, Paul. 1993. Adaptation and the goals of evolutionary research. The Quarterly Review of Biology 68: 1-32.

Reiss, John. 2009. Not by design. Berkeley: University of California Press.

Rosenberg, Alexander. 1985. The structure of Biological Science. Cambridge, Mass.: Cambridge University Press.

—. 1994. Instrumental Biology. Chicago: University of Chicago Press.

—. 2006. Darwinian reductionism. Chicago: University of Chicago Press.

Rosenberg, Alexander y McShea, Daniel. 2008. Philosophy of Biology. New York: Routledge.

Ruse, Michael. 2003. Darwin and design. Cambridge, Mass.: Harvard University Press.

- 2008. Charles Darwin. Buenos Aires: Katz.

Sober, Elliott. 1984. The nature of selection. Chicago: University of Chicago Press.

—. 1993. Philosophy of Biology. Oxford: Oxford University Press.

-. 2006. The two faces of fitness. En Conceptual issues in Evolutionary Biology, $3^{\circ}$ edition, ed. Elliott Sober, 25-38. Cambridge, Mass.: MIT Press.

Sterelny, Kim y Griffiths, Paul. 1999. Sex and Death. Chicago: Chicago University Press.

Wainwright, Stephen; Biggs, William; Currey, John y Gosline, Jeffrey. 1980. Diseño mecánico en organismos. Madrid: Blume.

Weibel, Ewald; Taylor, Richard y Bolis, Liana. 1998. Principles of animal design. Cambridge, Mass: Cambridge University Press.

West-Eberhard, Mary. 1998. Adaptation: current usages. En Philosophy of Biology. eds. David Hull y Michael Ruse, 8-14. Philosophy of Biology. Oxford: Oxford University Press.

Williams, George. 1966. Adaptation and Natural Selection. Princeton: Princeton University Press.

Gustavo Caponi es Doctor en Lógica y Filosofía de la Ciencia (UNICAMP, 1992), Profesor del Departamento de Filosofía de la Universidade Federal de Santa Catarina y becario del CNPq. Es autor de: Georges Cuvier: un fisiólogo de museo (UNAM: México, 2008); Buffon (UAM: México 2010); La segunda agenda darwiniana: contribución preliminar a una historia del programa adaptacionista (Centro de Estudios Filosóficos, Políticos y Sociales Vicente Lombardo Toledano: México, 2011); y Função e desenho na biologia contemporânea (Editora 34: São Paulo, 2012).

AdDRESS: Rua Esteves Jr. 605 (Apto.1414), 88015-130 Florianópolis SC, Brasil.

E-Mail: gustavoandrescaponi@gmail.com 